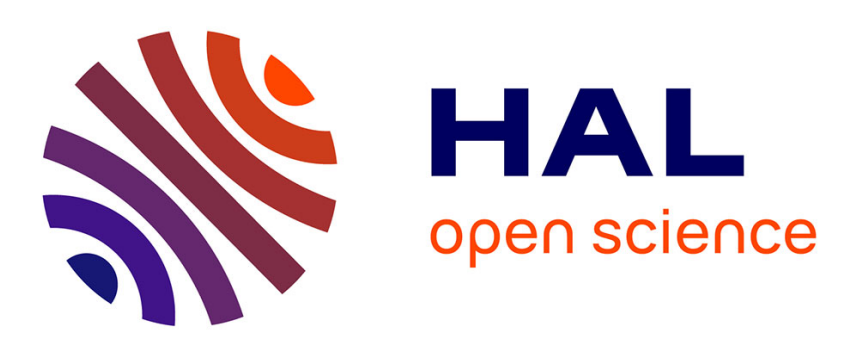

\title{
Polynomial time algorithms for constant capacitated single-item lot sizing problem with stepwise production cost
}

Ayse Akbalik, Christophe Rapine

\section{- To cite this version:}

Ayse Akbalik, Christophe Rapine. Polynomial time algorithms for constant capacitated single-item lot sizing problem with stepwise production cost. Operations Research Letters, 2012, 40, pp.390-397. 10.1016/j.orl.2012.05.003 . hal-00766771

\section{HAL Id: hal-00766771 https://hal.science/hal-00766771}

Submitted on 18 Dec 2012

HAL is a multi-disciplinary open access archive for the deposit and dissemination of scientific research documents, whether they are published or not. The documents may come from teaching and research institutions in France or abroad, or from public or private research centers.
L'archive ouverte pluridisciplinaire HAL, est destinée au dépôt et à la diffusion de documents scientifiques de niveau recherche, publiés ou non, émanant des établissements d'enseignement et de recherche français ou étrangers, des laboratoires publics ou privés. 


\title{
Polynomial time algorithms for constant capacitated single-item lot sizing problem with stepwise production cost
}

\author{
Ayse Akbalik*, Christophe Rapine \\ LGIPM, Université de Lorraine, Ile du Saulcy, 57045 Metz, France
}

\begin{abstract}
This paper presents two polynomial time algorithms for the constant capacitated lot sizing problem with a batch production. We give several optimality properties for the general problem. Assuming constant production capacity, constant batch size and WagnerWhitin cost structure, we derive $O\left(T^{4}\right)$ and $O\left(T^{6}\right)$ time algorithms respectively for the case with production capacity multiple of the batch size and for the case with arbitrary fixed capacities.
\end{abstract}

Keywords: Single-item, capacitated lot sizing problem, stepwise costs, polynomial time algorithm, dynamic programming.

\section{Introduction}

We study an extension of the well known single-item capacitated lot sizing problem (CLSP) considering fixed cost per batch in addition to a fixed setup cost. Classical CLSP deals with defining the optimal production quantities in order to satisfy the customer demand while minimizing the total production and storage costs and respecting the production capacity. Demand is assumed to be known for each period over a finite time horizon $T$, and has to be satisfied without backlogging. The production cost function of the classical lot sizing problem is composed of a fixed cost (setup cost), a unit production and a unit holding cost per period. Single-item lot sizing problem arises in many production and inventory planning problems in practical situations and is also used to solve more complex systems. Despite the simplicity of its description, the computational complexity of CLSP depends on many parameters (see [1] and [2]).

In this paper we consider a CLSP with a batch production, which incurs a fixed cost per batch in addition to the setup cost. We assume that the batch size and the production capacity does not vary over the planning horizon. Notice that we authorize fractional batch production contrary to different studies in the literature assuming only full batch production (e.g. [3]). The fixed cost per batch can be encountered quite often in practice, for instance when a machine is to be setup for a batch production. The same problem can also be seen as an integrated production and transportation problem for a capacitated manufacturer replenishing a depot with capacitated vehicles in a serial supply chain. If no storage activity occurs at the manufacturer stage, fixed transportation cost per vehicle can be considered as fixed cost per batch, with the batch size equals to the vehicle capacity (see [4]).

\footnotetext{
${ }^{*}$ Corresponding author.

Email addresses: ayse.akbalik@univ-metz.fr (Ayse Akbalik), christophe.rapine@univ-lorraine.fr (Christophe Rapine)
}

Both production setup cost and fixed cost per batch can be aggregated, resulting in a discontinuous production cost with a stepwise structure. In literature, such a cost structure is called stair-case, multiple setup, stepwise or truckload discount cost. Our problem can be stated as "Single-item capacitated lot sizing problem with stepwise production costs", for short CLSP-SW (see [5] and [4]).

We provide a review of the relevant literature in Section 2. In Section 3, we present a mathematical formulation of the problem. In Section 4 we give some optimality properties, either arising from the literature or introduced by ourselves. In Section 5 two polynomial time algorithms are given for the constant CLSP-SW: an $O\left(T^{4}\right)$ time algorithm for the case with production capacity multiple of the batch size, and an $O\left(T^{6}\right)$ time algorithm for the general case, where production capacity is not assumed to be a multiple of the batch size. Section 6 reports some computational experiments. Finally in Section 7 we present some concluding remarks and perspectives.

\section{Relevant literature}

The seminal papers [6] and [7] can be cited as the first studies on the lot sizing problem. In [8], Florian and Klein characterize the extreme points of the feasible domain of the CLSP under concave cost functions, which will be detailed in Section 4. Baker et al. [9] study single-item CLSP and propose an optimal solution property which will be stated in Section 4. This property has been extended to the CLSP with non-increasing unit production cost in [2]. In the following, we present relevant studies on the single-item lot sizing problem, with piecewise (or stepwise) costs and/or limited capacity, focusing on exact polynomial time approaches. This part is split into three categories: Uncapacitated case with stepwise cost (see Table 1); Constant capacitated case without stepwise cost (see Table 2) and Capacitated case with stepwise cost (see Table 3). Our contribution in the literature is clearly stated compared with those studies. 
As far as we know, one of the first studies on the integration of fixed transportation costs into the inventory control policy is performed by Lippman [10]. The author proposes an $O\left(T^{5}\right)$ algorithm for the single-item uncapacitated lot sizing problem with multiple-batch size production and without backlogging. For the same problem, Pochet and Wolsey [11] proposes an $O\left(T^{2} \min (T, P)\right)$ algorithm, with $B$ the batch size, improving that of [10]. Lee [12] studies a similar structure, but, in addition to the fixed cost per batch (or freight), the author considers also a setup cost for production. Observe that we consider in this paper the capacitated version of this problem. Several authors consider the so-called Wagner-Whitin (WW) cost structure. Recall that under WW cost structure, producing and storing one unit in period $t$ costs more than producing it later. Assuming WW cost structure, Lee [12] gives an $O\left(T^{4}\right)$ algorithm which is improved by Lee et al. [13] to $O\left(T^{3}\right)$ for a more general case with two-echelon structure and backlogging. For the latter problem with backlogging, Li et al. [3] proposes an $O\left(T^{3}\right)$ algorithm, using Monge matrices. The same authors extend their algorithm to a more general structure with concave cost functions and propose an $O\left(T^{3} \log (T)\right)$ algorithm. Under stochastic environment the reader is referred to [14] where a $O\left(T B^{3}\right)$ time algorithm is developed for the stepwise lot sizing problem with stochastic lead times.

Table 1: Results for uncapacitated problem with stepwise cost. In the assumptions column, $p_{t}^{f}$ refers to the setup production cost, $p_{t}^{b}$ to the fixed cost per batch, $B$ to the constant batch size and WW to the Wagner-Whitin cost structure.

\begin{tabular}{cc|c}
\hline & Assumptions & Uncapacitated, Stepwise \\
\hline \multirow{3}{*}{ No Backlog } & no setup cost, $p_{t}^{b}$ & {$[10], O\left(T^{5}\right)$} \\
& no setup cost, $p_{t}^{b}$ & {$[11], O\left(T^{2} \min (T, B)\right)$} \\
& $p_{t}^{f}, p_{t}^{b}, \mathrm{WW}$ & {$[12], O\left(T^{4}\right)$} \\
\hline Backlog & $p_{t}^{f}, p_{t}^{b}, \mathrm{WW}$ & {$[3]$ and $[13], O\left(T^{3}\right)$} \\
& $p_{t}^{f}, p_{t}^{b}$ & {$[3], O\left(T^{3} \log (T)\right)$} \\
\hline
\end{tabular}

All previous results consider the uncapacitated version of the problem. When dealing with the capacitated version, most of the authors assume a constant production capacity. For CLSP with constant production capacity, without backlogging and without multiple setup cost (i.e. without fixed cost per batch), Florian and Klein [8] give an $O\left(T^{4}\right)$ time algorithm which is improved by van Hoesel and Wagelmans [15] to $O\left(T^{3}\right)$ for more general concave production costs. For the same constant capacitated case without multiple setup, Pochet and Wolsey [11] solve also the problem in $O\left(T^{3}\right)$ time under linear cost assumptions. The reader can refer to [16] for a detailed presentation on the mixed integer programming approach to solve different extensions of CLSP.

Table 2: Results for constant capacitated case without stepwise cost and without backlogging.

\begin{tabular}{c|c}
\hline Assumptions & Constant capacity, No stepwise \\
\hline Concave production cost & {$[8], O\left(T^{4}\right)$} \\
Concave production cost & {$[15], O\left(T^{3}\right)$} \\
Linear costs & {$[11], O\left(T^{3}\right)$} \\
\hline
\end{tabular}

For the more general case studied in this paper, with both a limitation on the production capacity and a stepwise cost structure, we have only found the study of Van Vyve [5] in the literature. In [5], the author proposes an $O\left(T^{3}\right)$ algorithm for the single-item CLSP with multiple setups and backlogging. The author makes linear cost assumptions for each cost component. For the case without backlogging, an $O\left(T^{2} \log (T)\right)$ algorithm is proposed. Observe that in a sense his model is more general, for he considers time dependent production capacity expressed as the maximum number of installable batches per period, while we restrict to a constant production capacity. However there are other significant differences between our assumptions and those made in [5]. We point them below and outline the contributions of our paper:

1. We assume both classical setup cost of production and fixed cost per batch in our model, while Van Vyve [5] assumes only the fixed cost per batch. Observe that introducing setup costs to the model of Van Vyve renders the problem NP-hard. The reduction is immediate from the result of Florian et al. [1] considering null fixed cost per batch. The result still holds even for instances with unitary setup cost, no holding cost and stationary demands over time.

2. While the time dependency of the maximum number of installable batches makes the problem with setup cost NPhard, we prove in this paper that the problem becomes polynomial for a constant production capacity.

3. In [5], the maximum numbers of installable batches are non negative integers. For our model, this is equivalent to assume that production capacity $P$ is a multiple of batch size $B$. In this paper both cases are considered: $P$ $\bmod B=0$ (for which an algorithm in $O\left(T^{4}\right)$ is given) and $P \bmod B \neq 0$ (for which an algorithm in $O\left(T^{6}\right)$ is given).

As a consequence, our result establishes that the CLSP-SW with constant production capacity and constant batch size is polynomially solvable with the addition of a setup cost even for the case with $P \bmod B \neq 0$. We propose two polynomial time algorithms which are both original and represent a new contribution in the literature. To derive these algorithms we also assume linear costs with WW cost structure as in [5]. Table 3 is given to compare the complexity results of both paper.

Table 3: Results for capacitated case with stepwise costs, linear costs and con$\underline{\text { stant batch size } B \text {. }}$

\begin{tabular}{cc|c}
\hline & Other assumptions & Capacitated, Stepwise \\
\hline No Backlog & no setup cost, $p_{t}^{b}, \mathrm{WW}, P_{t} \bmod B=0$ & {$[5], O\left(T^{2} \log (T)\right)$} \\
No Backlog & constant capacity, $p_{t}^{f}, p_{t}^{b}, \mathrm{WW}, P \bmod B=0$ & Our study, $O\left(T^{4}\right)$ \\
No Backlog & constant capacity, $p_{t}^{f}, p_{t}^{b}, \mathrm{WW}$ & Our study, $O\left(T^{6}\right)$ \\
\hline Backlog & no setup cost, $p_{t}^{b}, \mathrm{WW}, P_{t} \bmod B=0$ & {$[5], O\left(T^{3}\right)$} \\
\hline
\end{tabular}

\section{Notation, Problem formulation}

In this section a general description of the problem is given. Later in the paper restrictive assumptions will be made on costs and capacities in order to derive polynomial time algorithms. 
Recall that the problem consists in planning a single-item production to meet discrete and deterministic demand $d_{t}$ over a finite time horizon $t \in\{1, \ldots, T\}$. We consider a production capacity $P_{t}$ in period $t$ at the plant, where a batch production takes place. The batch size is denoted by $B_{t}$ for each period $t$. The aim is to propose a feasible production planning (respecting capacity limits) which satisfies demand without backlogging, such that the total cost of production and storage is minimized.

A production plan $x$ is a vector of size $T$ corresponding to the amount $x_{t}$ to produce each period. Hence a plan $x$ is feasible iff (i) $0 \leq x_{t} \leq P_{t}$ and (ii) $\sum_{u=1}^{t} x_{u} \geq \sum_{u=1}^{t} d_{u}$ for all $t \in\{1, \ldots, T\}$. We consider the following cost structures:

Production. Production cost $p_{t}(a)$ of producing the amount $a$ at period $t$ includes a discrete and a continuous part. The discrete part is composed of a setup cost $p_{t}^{f}$ paid whatever the amount $a>0$ and of a fixed cost per batch $p_{t}^{b}$ paid for each batch produced. We call variable production cost the continuous part $p_{t}^{u}$ of the production cost, which is assumed to be concave with $a$. We have the following expression of the production cost:

$$
p_{t}(a)=\mathbf{1}_{\{a>0\}} p_{t}^{f}+p_{t}^{b}\left\lceil\frac{a}{B_{t}}\right\rceil+p_{t}^{u}(a)
$$

Notice that $p_{t}$ appears as a function on $\left[0, B_{t}\right]$ repeated with a vertical step of $p_{t}^{b}$ every $B_{t}$ units. The resulting function is not concave even if $p_{t}^{u}$ is, but stepwise concave, possibly with discontinuity every $B_{t}$ units. When the variable production cost $p_{t}^{u}$ is linear, $p_{t}$ is said to be stepwise linear. We also assume that $B_{t}$ is lower than production capacity $P_{t}$. For the case with constant production capacity and constant batch size, if $B \geq P$, the problem is known to be polynomial (see [1]).

In what follows, we first restrict our attention to concave costs to use some optimality properties proposed in the literature. Then, we restrict further to stepwise linear costs in order to derive polynomial time algorithms.

Storage. To a plan $x$, one can associate the auxiliary vector $s=\left(s_{1}, \ldots, s_{T}\right)$ where $s_{t}$ denotes the inventory level at the beginning of period $t$. The classical material balance equation writes down as $s_{t+1}=s_{t}+x_{t}-d_{t}$. We consider wlog that initial and final inventory levels are zero $\left(s_{1}=s_{T+1}=0\right)$. Thus, condition (ii) can be replaced by (ii') $s_{t} \geq 0$. There is no capacity limit on the inventory level. The cost to keep quantity $s$ in stock from period $t-1$ to $t$ is $h_{t}(s)$. We assume $h_{t} \geq 0$.

The problem is then to find a production plan $\left(x_{1}, \ldots, x_{T}\right)$ minimizing:

$$
\sum_{t=1}^{T} p_{t}\left(x_{t}\right)+h_{t}\left(s_{t}\right)
$$

respecting the production capacity :

$$
0 \leq x_{t} \leq P_{t} \forall t=1, \ldots, T
$$

and the classical inventory flow conservation :

$$
\begin{array}{ll}
s_{t+1}=s_{t}+x_{t}-d_{t} & \forall t=1, \ldots, T \\
s_{1}=s_{T+1}=0 & \forall t=1, \ldots, T \\
s_{t} \geq 0 &
\end{array}
$$

We will now introduce some dominance properties for the purpose of developing polynomial time algorithms under various assumptions.

\section{Optimality properties}

Dynamic programming approaches for lot sizing are based on decomposition properties on the inventory level in order to apply the sub-optimality principle of Bellman. Indeed, if in a certain optimal production schedule the inventory level has a value of $s$ in period $t$, the problem can be decomposed into two parts: Finding the best planning between 1 and $t$ with a final inventory level $s$, and finding the best planning between $t$ and $T$ with an initial inventory level $s$. In this setting, regeneration points introduced in [17] play a central part (see this definition in [8]). For a given planning, a period $t$ is said to be a regeneration point if its initial inventory is zero, i.e. $s_{t}=0$. Florian and Klein [8] define a subplan as a sub-sequence of production between two consecutive regeneration points.

Definition 1 (Subplan, [8]). Given a planning, a subsequence of production on periods $u, \ldots, v-1$ is a subplan, denoted $S_{(u v)}$, if $s_{u}=s_{v}=0$, and $s_{t}>0$ for all $t=u+1, \ldots, v-1$.

Notice that at least 2 regeneration points exist in any planning, since $s_{1}=s_{T+1}=0$. In [8], the authors use the notion of subplans to decompose the time horizon into sub-sequences which start and end with zero stock. The optimum can then be computed in $O\left(T^{2}\right)$ as a shortest path problem, given the costs of the $O\left(T^{2}\right)$ possible subplans. To efficiently compute the optimal planning on a subplan, the following definition is introduced in [8]:

Definition 2 (Capacity constrained subplan, [8]). A subplan is capacity constrained if it contains at most one period with a production neither null nor at production capacity.

Assuming concave unit production costs, the following characterization property is proven in [8] :

Property 1 ([8]). When cost functions are concave, a planning belongs to the set of extreme points of solutions if and only if it can be decomposed into capacity constrained subplans.

This property can be seen as a generalization of the ZIO dominance property used by Wagner and Whitin [7] to design their algorithm. Recall that ZIO (Zero Inventory Ordering) policies consist in ordering only when inventory level drops to zero. A similar property has also been stated by Lippman [10] as regeneration point property. Notice that Florian and Klein [8] consider no stepwise production cost, contrary to Lippman [10] who in turn considers no production setup cost. We now demonstrate with a simple example that Property 1 does not hold when considering stepwise production costs.

Example 1. Consider a time horizon of 3 periods with demands $(1,2,3)$ to satisfy. Production capacity is 3 and batch 

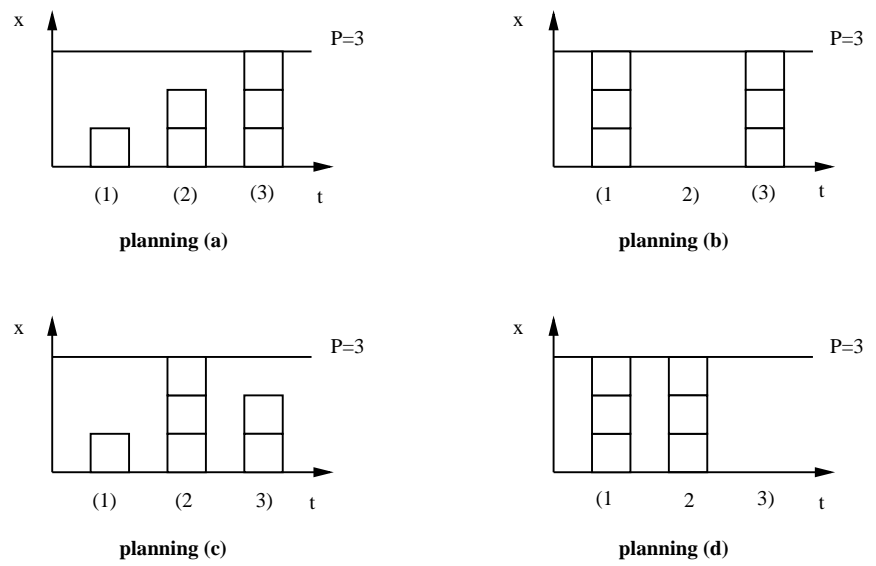

Figure 1: Best capacity constrained planning for each subplan decomposition in Example 1.

size is 2 units. Set-up costs are set to 2 for production, and 5 per batch. A unit holding cost of 0.5 is paid per product and per unit of time. Other costs are null.

Clearly there exist 4 possible subplan decompositions: (1)(2)(3), (1-2)(3), (1)(2-3), (1-2-3). In the first one, each period forms a subplan; in the second, the first two periods 1 and 2 constitute a subplan, etc. The best capacity constrained plannings associated to each decomposition are given in Figure 1. In fact there is only one possible capacity constrained schedule for each decomposition. Their costs are respectively: (a) 26, (b) 25, (c) 26.5, (d) 26.5. Hence the best planning respecting the capacity constrained property for the instance is $(b)$. However the optimal planning consists of producing 2 units at each period, resulting in a cost of 22. This example shows that capacity constrained subplans are not dominant with stepwise linear production costs.

Notice that in the example, the optimal solution has only one subplan, but with 3 periods with production not at full capacity. With stepwise production costs, Property 1 is not verified any more due to the fact that it can be preferable to produce only full batch sizes to save fixed cost per batch instead of saturating the production capacity.

For the CLSP with stepwise costs, we introduce the following definitions on production levels:

Definition 3. For a given planning, a period t is said to be:

- $\boldsymbol{P}$-saturated if production is at full capacity $\left(x_{t}=P_{t}\right)$. In the literature "full-truck load period" is also used concerning similar dispatch scheduling problems.

- B-saturated if production corresponds to the maximum number of full batches, without any fractional batch $\left(x_{t}=\right.$ $\left.\left\lfloor P_{t} / B_{t}\right\rfloor B_{t}\right)$

- Fractional, if there exists a non-complete batch ( $x_{t} \bmod$ $B_{t} \neq 0, x_{t}>0$ and $\left.x_{t}<P_{t}\right)$. For the same concept, lessthan-truckload period is also used in the literature.
- FBS (Full Batch Size) finally if the period is neither Psaturated, B-saturated, null (zero production) nor fractional. This corresponds to a production of only full batches $\left(x_{t} \bmod B_{t}=0\right)$, but not B-saturated.

Let us remark that in a fractional period an empty space remains in a batch as well as an unused production capacity. Hence, at least one more unit can be produced with no additional fixed cost. This situation should be quite rare in an optimal schedule, which is expressed formally in Property 2. First, we introduce the following definition which generalizes Definition 2:

Definition 4 (Batch Constrained Subplan). A subplan is batch constrained if it contains at most one fractional period.

The following property by Lee et al. [13] generalizes the property in [10] which assumes only fixed transportation costs (in CLSP-SW, fixed transportation cost corresponds to the fixed cost per batch). It stands that between two consecutive regeneration points, there is at most one fractional batch production.

Property 2 (Decomposition Property, [13]). There exists an optimal planning which can be decomposed into batch constrained subplans for stepwise concave costs. Thus, there exists at most one fractional period in each subplan.

We now introduce a dominance property for the capacitated case, inspired by Baker et al. [9], who states that there exists an optimal planning such that for each period $t, s_{t}\left(P_{t}-x_{t}\right) x_{t}=0$. This property means that if the inventory level at the beginning of period $t$ is strictly positive, the production is either null or at maximum capacity. In [9], this property is proven with time varying production capacity and setup costs, but stationary linear production and holding costs. Bitran and Yanasse [2] have extended this result to linear functions non-increasing over time. We generalize this dominance to stepwise linear functions (linearity is based on the quantities) under WW cost structure:

Property 3. If the variable production cost $p_{t}^{u}$ is linear in the quantity produced and follows WW cost structure, then there exists an optimal schedule which verifies for each period $t$

$$
s_{t}\left(P_{t}-x_{t}\right)\left(x_{t} \bmod B_{t}\right)=0
$$

As a consequence, if the inventory level is strictly positive, period $t$ cannot be a fractional period. Hence a fractional period can only appear as the first period of a subplan.

Proof. We will show how to transform an arbitrary planning into a planning of lower cost which verifies the property. Consider an optimal planning with $t$ the latest period such that $s_{t}>0, x_{t} \bmod B_{t} \neq 0$, and $x_{t}<P_{t}$. Let us write $q=x_{t} \bmod B_{t}$ the fractional batch size. Consider one unit in stock at the beginning of period $t$. This unit has been produced at the first preceding period $t^{\prime}$ such that $x_{t^{\prime}}>0$ (we can assume that the demand is satisfied using the first-in-first-out principle, i.e. production at a period is assigned to the first unsatisfied demand). Notice that, inventory level can only decrease between $t^{\prime}$ and $t$. 
If we transfer this production unit from $t^{\prime}$ to $t$, then the resulting planning remains feasible: stock levels are positive or null, and production capacity at $t$ is necessarily respected ( $t$ was not P-saturated). When we compare costs, no additional setup cost appears, since a setup for production and for batch is already paid at $t$ for $q$. The variable part can only decrease due to the assumption of WW cost structure.

Hence, we have obtained a better planning. We can then repeat this transformation, till the planning becomes infeasible or a setup cost appears. This corresponds to delay an amount of $\min \left\{B_{t}-q, P_{t}-q, s_{t}\right\}$ at period $t$. In each case the new schedule verifies the property for period $t$.

Delaying the production of a part of $s_{t}$ at period $t$ may create new periods which do not satisfy Property 3. However such periods can only appear before $t$. We can therefore repeat this process on the new latest fractional period with positive stock, till the beginning of the planning is reached. It shows that we can transform any schedule to verify the property on a sub-sequence $1, \ldots, t$ without modifying the end of the schedule on $t+1, \ldots, T$. We will use this fact for proof of Property 4. $\square$

Notice that until now, we only require the variable production cost to have WW cost property, without making any assumption on the fixed cost per batch. Indeed no new setup cost is involved in the transformation. As a corollary, if the fixed cost per batch also has WW cost structure, and the batch sizes are stationary, we have the following property:

Property 4. In addition to Property 3, there exists an optimal schedule which verifies for each period $t$,

$$
s_{t} \geq B \Rightarrow x_{t}\left(P_{t}-x_{t}\right)\left(\left\lfloor P_{t} / B\right\rfloor B-x_{t}\right)=0
$$

The property implies that if the inventory level is higher than the batch size, then the production can only be null, P-saturated or B-saturated.

Proof. We consider an optimal planning verifying the Property 3. Again, we use a simple interchange argument. Consider the latest period $t$ of a schedule which does not verify the implication: $s_{t} \geq B$ while the production at period $t$ is neither null, nor saturated. As inventory level is strictly positive and the production is not $\mathrm{P}$-saturated, $t$ must be an FBS period due to Property 3 . Let $t^{\prime}$ be the last period preceding $t$ where production occurs $\left(x_{t^{\prime}}>0\right)$. We now show that we can delay a full batch from $t^{\prime}$ to $t$ without increasing the cost of the planning.

First notice that at least $B$ units are produced at time $t^{\prime}$. Indeed, if inventory level $s_{t^{\prime}}$ is strictly positive, Property 3 again imposes that at least a full batch size is produced. Otherwise $s_{t^{\prime}}=0$ and we necessarily have $x_{t^{\prime}}=d_{t^{\prime}}+\cdots+d_{t}+s_{t} \geq s_{t} \geq B$.

We can therefore delay $B$ units from $t^{\prime}$ to $t$ maintaining the feasibility of the schedule: inventory levels between $t^{\prime}$ and $t$ are greater than $s_{t}$ and hence greater than $B$, and an additional batch can always be produced in an FBS period without violating production capacity constraint. This interchange can only decrease the cost of the schedule, due to the WW cost assumptions on the fixed cost per batch: it is always more economical to delay the production of a full batch size to a period whose setup production cost is already paid.

Notice that, as exactly a quantity $B$ has been delayed, period $t$ remains an FBS period, and thus still fulfills Property 3. It may be a different matter for period $t^{\prime}$ if it was P-saturated in the planning. However, in this case we can modify the new schedule only on sub-sequence $1, \ldots, t^{\prime}$ such that it verifies Property 3 (cf the proof of this property).

Repeating the transformation leads to either a B-saturated period at $t$, or to a drop in the inventory level $s_{t}$ below $B$, verifying the property at $t$. We then iterate the same process on previous periods till the beginning of the schedule is reached.

In order to efficiently detect FBS periods in a subplan, we introduce the notion of sub-interval. Let us assume that the batch size is stationary, $B_{t}=B$. As a result of Property 4 , the periods with an inventory level lower than $B$ play a particular role. This role is analogous to that of regeneration points when no fixed costs per batch are involved. Indeed only 3 production possibilities exist for other periods with an inventory level greater or equal than $B$, namely $0, P_{t}$ and $\left\lfloor P_{t} / B\right\rfloor B$. We define, similarly to Definition 1, a sub-interval as follows:

Definition 5 (Sub-interval). Given a planning, $a$ subsequence of production on periods $k, \ldots, f-1$ is a sub-interval, denoted $S_{[k f]}$, if $s_{k}$ and $s_{f}$ are strictly lower than $B$, and $s_{t} \geq B$ for all $t=k+1, \ldots, f-1$.

Notice that a sub-interval is necessarily included in a subplan. We denote a sub-interval $S_{(u f]}$ when inventory level is null at $u$, i.e. $u$ is the begining of the subplan. The same notation stands for $S_{[k v)}$ with $v$ the end of a subplan.

In the remaining of the paper we make the following assumptions, implying in particular that Property 4 holds:

- Batch size $B_{t}=B$ is stationary.

- Production cost $p_{t}$ is a stepwise linear function of the quantity produced, for each period $t$. Moreover, the variable production cost $p_{t}^{u}$ and the fixed cost per batch $p_{t}^{b}$ have WW cost structure. Fixed production cost $p_{t}^{f}$ can take any positive values.

Under these assumptions, we have the following corollary of Property 4, underlying the similarity between subplan and subinterval:

Corollary 1 (Fractional \& FBS periods). Fractional periods can only take place at the beginning of a subplan, while FBS periods can only take place at the beginning of a sub-interval.

In the next section, under these assumptions, we present a polynomial time algorithm to find an optimal planning, first in the case where $P \bmod B=0$, second for any constant values of $P$ and $B$. 


\section{Polynomial time algorithms}

The main idea of both algorithms is to compute the minimum $\operatorname{cost} Z^{*}(u, v)$ associated to each possible batch constrained subplan $S_{(u, v)}$. Using the decomposition property 2 , the cost of an optimal schedule can then be computed in $O\left(T^{2}\right)$ by a shortestpath like dynamic programming algorithm. The computation of the optimum cost of a subplan relies on the notion of subinterval, and the computation of the cost of sub-intervals constitutes hence the heart of our approach. Let $u$ and $v$ be two consecutive regeneration points, and $S_{[k f]}$ a sub-interval inside this subplan. Let us denote by $C^{v}[k, f]$ the cost of the optimal planning on the sub-interval $S_{[k f]}$, assuming $v$ as the next regeneration point. This cost includes production cost inside $[\mathrm{k}, \mathrm{f}]$ and inventory holding costs till the beginning of period $f$.

We first present the cost computation for the case where $P$ is multiple of $B$. We describe the production structure of a constant production capacitated case and we give the idea of the solution algorithm. In the next section we extend this result to the general case.

Figure 2 gives the production structure of a batch constrained subplan, based on Properties 3 and 4: inside a sub-interval, only saturated periods can be encountered. The entering inventory

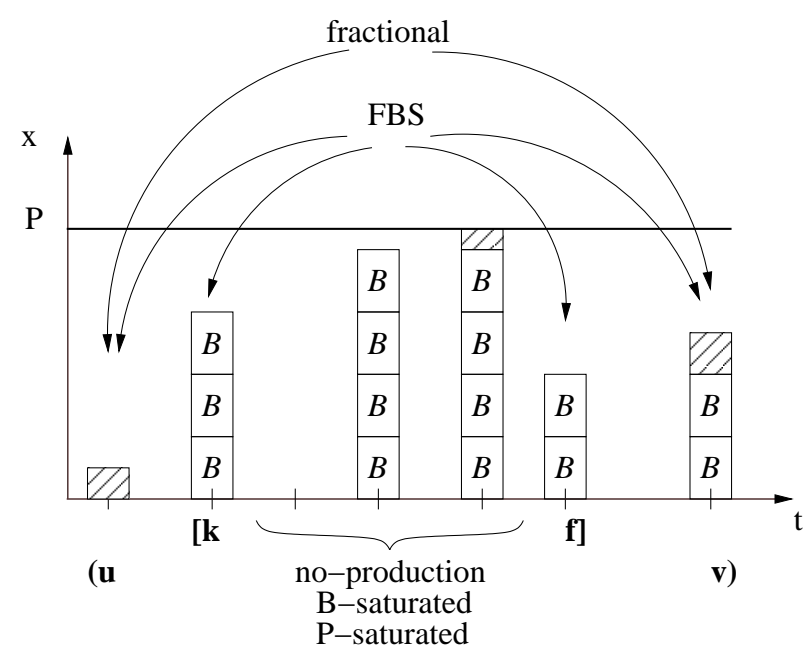

Figure 2: Structure of a batch constrained subplan with stationary production capacity $P$ (where $P$ does not necessarily divide $B$ ). In the figure, $(u v)$ denotes a subplan and $[k f]$ a sub-interval. We represent the different types of production that are possible at each period.

level $\widehat{s}_{k}^{v}$ and the production $\widehat{x}_{k}$ at the beginning of a sub-interval depend on the number of P-saturated and B-saturated periods in the subplan. We use the following notations:

$\rho_{[a, b]}:$ number of $\mathrm{P}$-saturated periods on sub-sequence $a, \ldots, b-1$ for a given schedule.

$\vartheta_{[a, b]}:$ number of B-saturated periods on sub-sequence $a, \ldots, b-1$ for a given schedule.

We also denote by $Q=\lfloor P / B\rfloor B$ the production quantity of a B-saturated period.

\subsection{Case with $P$ multiple of $B$}

In this case $\mathrm{P}$-saturated and $\mathrm{B}$-saturated periods coincide and we have $Q=P$. As a consequence, all production quantities inside a subplan are multiple of the batch size. The expression of the inventory level $\widehat{s}_{k}^{v}$ represents exactly the total demand until the next regeneration point modulo $B$. However the production quantity at period $k$ depends on the number of B-saturated periods in the sub-interval. Using the inventory flow conservation on $S_{[k f]}$, we have:

$$
x_{k}+\vartheta_{[k+1, f]} Q=\sum_{t=k}^{f-1} d_{t}+s_{f}-s_{k}
$$

Let us remark that the number $\vartheta_{[k+1, f]}$ of B-saturated periods is (almost) fixed for given inventory levels $s_{f}$ and $s_{k}$. Indeed let $(q, r)$ be the quotient and the rest of the Euclidean division of the right side of the previous equation by $Q$. If $x_{k}<Q$, we have by definition $\left(\vartheta_{[k+1, f]}, x_{k}\right)=(q, r)$. Otherwise we have $x_{k}=Q$, $r=0$ and $\vartheta_{[k+1, f]}=q-1$. Hence the number $\widehat{\vartheta}_{[k f]}$ in a dominant schedule is given by $\left\lfloor\left(\sum_{t=k}^{f-1} d_{t}+\widehat{s}_{f}^{v}-\widehat{s}_{k}^{\nu}\right) / Q\right\rfloor$. We can state the following proposition:

Proposition 1. The production at the beginning of a subinterval $S_{[k f]}$, included in a subplan $S_{(u v)}$, depends only on periods $f$ and $v$, and is given by

$$
\begin{aligned}
\widehat{x}_{k} & =\left\{\begin{array}{l}
\left(\sum_{t=k}^{f-1} d_{t}+\widehat{s}_{f}^{v}-\widehat{s}_{k}^{v}\right) \bmod Q \text { if this quantity is not null } \\
0 \text { or } Q \text { otherwise (no production or } B \text {-saturated period) }
\end{array}\right. \\
\text { with } \widehat{s}_{k}^{v} & =\left\{\begin{array}{l}
0 \text { if } k=u(k \text { is the beginning of the subplan) } \\
\left(\sum_{t=k}^{v-1} d_{t}\right) \bmod B \text { otherwise }
\end{array}\right.
\end{aligned}
$$

In addition we must have $\widehat{x}_{k}+\widehat{s}_{k}^{v} \geq d_{k}$ for the planning to be feasible.

The entering inventory of a sub-interval is entirely determined by the next regeneration point. To compute dynamically $Z^{*}(u, v)$, we introduce $Z^{*}[k, v)$ the cost of an optimal planning between the first period $k$ of a sub-interval and $v$, the next regeneration point. If we know that the sub-interval ends at period $f$ (possibly $f=v$ ), since the planning is fixed by $v$ on $S_{[k f]}$, Bellman's sub-optimality principle applies and we have $Z^{*}[k, v)=C^{v}[k, f]+Z^{*}[f, v)$. Figure 3 gives a picture of these different notations. Hence we can compute dynamically $Z^{*}[k, v)$ based on the recursive equation:

$$
Z^{*}[k, v)=\min \left\{C^{v}[k, f]+Z^{*}[f, v) \mid k<f \leq v\right\}
$$

The different steps of the computation of $Z^{*}(u, v)$ are summarized in pseudo-code in Algorithm 1. In the last step, the cost of an optimal schedule $O P T[u]$ on sub-sequence $u, \ldots, T+1$ is dynamically computed as a shortest path in the graph where nodes are the regeneration points and arcs are weighted with the $\operatorname{cost} Z^{*}(u, v)$ to pass from node $u$ to node $v$.

Let us see how to compute the $\operatorname{cost} C^{v}[k, f]$ of sub-intervals. Notice that with the possibility of having production inside the 


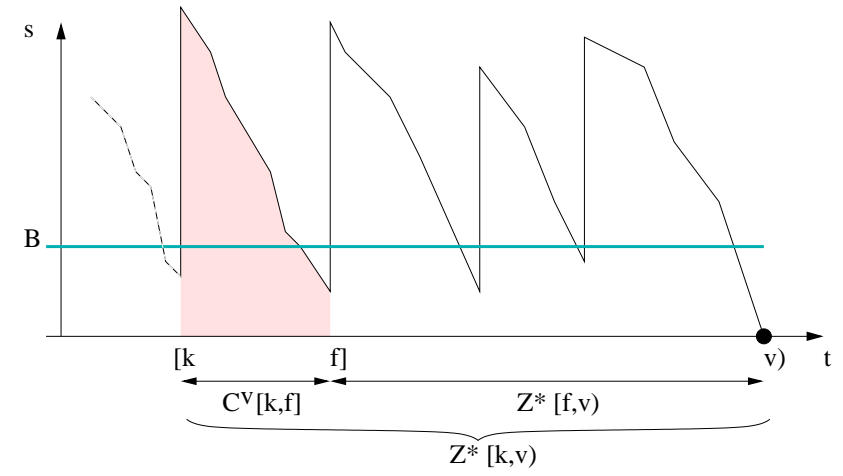

Figure 3: Dynamic computation of $Z^{*}[k, v)$

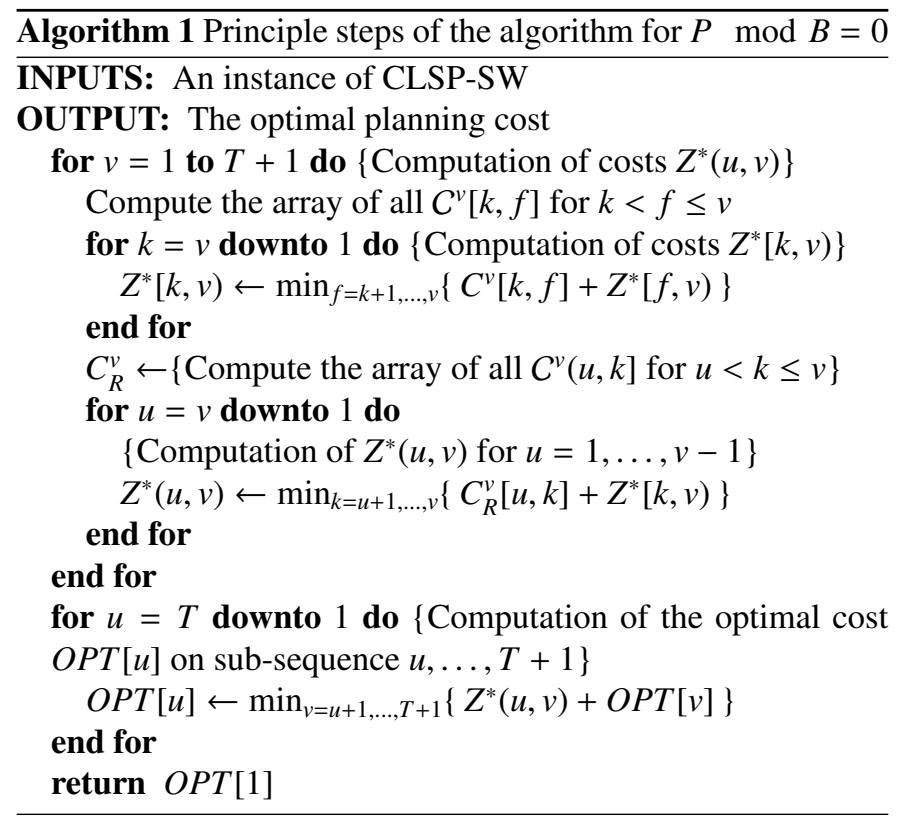

sub-interval, the inventory level at a period $t(k<t \leq f \leq$ $v$ ) depends on the number of saturated periods between $t$ and $f$. Let us denote by $\tilde{s}_{t}(\vartheta)$ the entering inventory level at $t$ if exactly $\vartheta \mathrm{B}$-saturated periods occur in $t, \ldots, f-1$. Inventory flow conservation gives:

$$
\tilde{s}_{t}(\vartheta)=\sum_{i=t}^{f-1} d_{i}+\widehat{s}_{f}-\vartheta Q
$$

Let us introduce $G(t, \vartheta)$ the minimum cost of a planning between the end of period $t$ till the beginning of period $f$, such that exactly $\vartheta$ B-saturated periods occur in sub-sequence $t+$ $1, \ldots, f-1$. The final inventory level at $f$ is $\widehat{s}_{f}$. In addition we require that the inventory level at each period is greater than $B$. Due to the dominances we clearly have

$$
C^{v}[k, f]=\min \begin{cases}p\left(\widehat{x}_{k}\right)+G\left(k, \widehat{\vartheta}_{[k, f]}\right) & \text { if } \widehat{x}_{k}<Q \\ p(Q)+G\left(k, \widehat{\vartheta}_{[k, f]}-1\right) & \text { if } \widehat{x}_{k}=Q\end{cases}
$$

For fixed $f$, the computation of all $C^{v}[k, f]$ can be achieved in linear time by a backward iteration, since quantities $\widehat{x}_{k}$ and $\vartheta_{[k f]}$ can be clearly deduced from their values at $k+1$ in constant time. This stands, of course, if $G(t, \vartheta)$ are precomputed first for all values $t=1, \ldots, f-1$ and $\vartheta=0, \ldots, f-t-1$. Since only 2 production levels are possible $(0$ or $Q)$, one can express recursively this quantity with the simple equation:

$G(t, \vartheta)=h_{t}\left(\tilde{s}_{t+1}(\vartheta)\right)+\min \left\{\begin{array}{l}G(t+1, \vartheta) \text { if } \tilde{s}_{t+1}(\vartheta) \geq d_{t+1} \\ / / \text { no production at } t+1 \\ p_{t+1}(Q)+G(t+1, \vartheta-1) \\ \text { if } \tilde{s}_{t+1}(\vartheta-1) \geq d_{t+1} \\ / / t+1 \text { is } B \text {-saturated } \\ +\infty \text { if } s_{t+1}(\vartheta)<B \text { and } \mathrm{t}+1<\mathrm{f} \\ / / \text { not valid }\end{array}\right.$

As initial conditions we have $G(f, 0)=0$ and $G(f, \vartheta)=+\infty$ for $\vartheta>0$. Since $\tilde{s}_{t}$ can be deduced in constant time from $\tilde{s}_{t+1}$, for given $f$ and $v$, one can compute $G$ for all $\vartheta$ and $\rho$ with a complexity in $O\left(T^{2}\right)$. It results that for a given regeneration point $v$, the computation of the cost of all the sub-intervals before $v$ takes a time complexity in $O\left(T^{3}\right)$ for a space in $O\left(T^{2}\right)$. The overall complexity of Algorithm 1 has a time complexity in $O\left(T^{4}\right)$ and an $O\left(T^{2}\right)$ space complexity.

\subsection{General Case}

We relax now the assumption that $P$ is multiple of $B$. In this case, the periods inside a sub-interval can be either with no production, B-saturated or $\mathrm{P}$-saturated. These latter periods $(P \neq Q)$ are not multiple of batch size $B$, and thus the entering inventory level of the sub-interval will depend on them. Let us denote by $\rho_{[k v)}$ the number of P-saturated periods on the subsequence $k, \ldots, v-1$. The inventory flow conservation can be written now as:

$$
\widehat{s}_{k}^{v}\left(\rho_{[k v)}\right)=\left\{\begin{array}{l}
0 \text { if } k=u(k \text { is the beginning of the subplan }) \\
\left(\sum_{t=k}^{v-1} d_{t}-\rho_{[k v)} P\right) \bmod B, \text { otherwise }
\end{array}\right.
$$

Consider a sub-interval $S_{[k f]}$ included in a subplan $S_{(u v)}$. Since inventory level at period $k$ is function of the number of Psaturated periods till next regeneration point, the cost $C^{v}[k, f]$ cannot be entirely determined by $v$. For given $\rho_{[k, v)}$ and $\rho_{[f v)}$, one can compute inventory levels $s_{k}$ and $s_{f}$ and thus decompose the problem into computing the best planning on the sub-interval respecting those inventory levels. Let us note $C^{v}[k, f]\left(\rho_{[k, f]}, \rho_{[f, v)}\right)$ the minimum cost of a schedule on the subinterval that admits exactly $\rho_{[k, f]}$ P-saturated periods on $S_{[k f]}$ and $\rho_{[f, v)}$ P-saturated periods on sub-sequence $f, \ldots, v-1$. In such a schedule, production at period $k$ corresponds to the total demand in sub-interval $S_{[k, f]}$ which is not satisfied by saturated periods (neither P-saturated nor B-saturated), knowing that the entering inventory is $\widehat{s}_{k}^{v}\left(\rho_{[k f]}+\rho_{[f, v)}\right)$ and the final inventory at $f$ is $\widehat{s}_{f}^{v}\left(\rho_{[f, v)}\right)$. With material balance constraint we have only 3 possibilities for $x_{k}$ :

$\widehat{x}_{k}=\left\{\begin{array}{l}P \quad(k \text { is P-saturated }), \text { or } \\ Q \quad(k \text { is B-saturated }), \text { or } \\ \left(\sum_{t=k}^{f-1} d_{t}+\widehat{s}_{f}^{v}\left(\rho_{[f, v)}\right)-\widehat{s}_{k}^{v}\left(\rho_{[k f]}+\rho_{[f, v)}\right)-\rho_{[k f]} P\right) \bmod Q\end{array}\right.$ 
We denote for short by $\widehat{\vartheta}_{k}$ the quotient in the Euclidian division of $\sum_{t=k}^{f-1} d_{t}+\widehat{s}_{f}^{v}\left(\rho_{[f, v)}\right)-\widehat{s}_{k}^{v}\left(\rho_{[k v)]}\right)-\rho_{[k f]} P$ by $Q$. Based on the 3 potential productions at $k$, we then have the formula:

$$
\begin{gathered}
C^{v}[k, f]\left(\rho_{[k, f]}, \rho_{[f, v]}\right)=p_{k}\left(\widehat{x}_{k}\right)+ \\
+\min \begin{cases}F\left(k, \widehat{\vartheta}, \rho_{[k f]}-1\right) & \text { if } \widehat{x}_{k}=P \\
F\left(k, \widehat{\vartheta}-1, \rho_{[k f]}\right) & \text { if } \widehat{x}_{k}=Q \\
F\left(k, \widehat{\vartheta}, \rho_{[k f]}\right) & \text { otherwise }\end{cases}
\end{gathered}
$$

where $F(t, \vartheta, \rho)$ is the minimum cost of a planning from the end of period $t$ till the beginning of period $f$, with no fractional nor FBS periods, but with exactly $\vartheta$ B-saturated periods and $\rho$ Psaturated periods. The inventory level at each period must be greater than $B$ and the final inventory at $f$ must be $\widehat{s}_{f}\left(\rho_{[f, v)}\right)$. Notice that the inventory level at period $t$ is hence equal to the quantity $\tilde{s}_{t}(\vartheta, \rho)=\sum_{i=t}^{f-1} d_{i}+\widehat{s}_{f}^{v}\left(\rho_{[f, v)}\right)-\vartheta Q-\rho P$. We have the following recursive expression for $F$ :

$$
\begin{gathered}
F(t, \vartheta, \rho)=h_{t}\left(s_{t+1}(\vartheta, \rho)\right)+ \\
+\min \begin{cases}F(t+1, \vartheta, \rho) & \text { if } \tilde{s}_{t+1}(\vartheta, \rho) \geq d_{t+1} \\
p_{t+1}(Q)+F(t+1, \vartheta-1, \rho) & \text { if } \tilde{s}_{t+1}(\vartheta-1, \rho) \geq d_{t+1} \\
p_{t+1}(P)+F(t+1, \vartheta, \rho-1) & \text { if } \tilde{s}_{t+1}(\vartheta, \rho-1) \geq d_{t+1} \\
+\infty \text { if } \tilde{s}_{t+1}(\vartheta, \rho)<B & \text { if } t+1<f\end{cases}
\end{gathered}
$$

For given $f, v$ and $\rho[f, v)$, computing $F$ for all possible triplets $(t, \vartheta, \rho)$ is achievable in time $O\left(T^{3}\right)$, since $\tilde{s}_{t}$ can be deduced in constant time from preceding values. Thus, computing all $F$ values is $O\left(T^{6}\right)$ time. The time complexity of the algorithm to compute $C^{v}[k, f]$ takes $O\left(T^{5}\right)$ time. Its aim is to compute all possible costs $C^{v}[k, f]\left(\rho_{[k, f]}, \rho_{[f, v]}\right)$ of sub-intervals for a given regeneration point $v$. The space complexity is simply in $O\left(T^{4}\right)$ to store each value.

The recursive formula to compute $Z^{*}[k, v)(\rho)$, which is the optimal planning cost over $k, \ldots, v-1$ with exactly $\rho \mathrm{P}-$ saturated periods is given by:

$$
Z^{*}[k, v)(\rho)=\min _{f} \min _{\rho_{[k f]}}\left\{C^{v}[k, f]\left(\rho_{[k, f]}, \rho-\rho_{[k, f]}\right)+Z^{*}[f, v)\left(\rho-\rho_{[k, f]}\right)\right\} .
$$

The computation of all these costs for a given $v$ can be done in space $O\left(T^{2}\right)$ and time $O\left(T^{4}\right)$, if costs $C^{v}[k, f]$ are provided. The optimal cost of the subplan $S_{(u v)}$ is then:

$$
Z^{*}(u, v)=\min _{\rho}\left\{Z^{*}(u, v)(\rho)\right\}
$$

which requires a complexity in $O\left(T^{2}\right)$ time to determine these values for given $u$ and $v$. In total, the algorithm has a time complexity in $O\left(T^{6}\right)$ for a space in $O\left(T^{4}\right)$.

\section{Computational experiments}

The aim of this section is to demonstrate the efficiency of our dynamic programming (DP) based algorithm versus a mixed integer linear programming (MILP) formulation solved via a commercial software. The MILP is coded in Mosel and executed with Xpress IVE version 1.22. The DP is coded in Java.
Tests are carried out on an Intel CORE, $2.70 \mathrm{GHz}$ with $4 \mathrm{~Gb}$ of RAM. In the literature there are different MILP formulations proposed for the lot sizing problem. We have chosen the aggregated formulation (AGG) since it is the most natural and has been shown to be quite efficient for the capacitated case (see [18]). In Xpress default parameters are used for cut strategy and presolving.

The complexity of the DP is only dependent on T, the length of the time horizon. In contrast the computational time of the MILP is very sensitive on each parameter: cost parameters, horizon length, demands, production capacity, batch size, etc. For a given instance, the resolution with no holding cost can be very hard to achieve for Xpress, whereas with a positive holding cost the resolution can be instantaneous. And vice versa. Our aim is not to conduct exhaustive experiments, but to demonstrate that ( $i$ ) our DP algorithm can be used on quite large practical instances, and (ii) the MILP can perform quite badly in regards to the computational time on this polynomial time problem. For this reason, we consider the following instance where all the parameters are stationary: $P=200, B=40, f=100$, $q=10, h=1, p=0$ and a demand of 41 units for each period. Notice that we are in the case $P \bmod B=0$. We carried out tests for different length of horizon, reported in Table 4. We limit the running time of Xpress to $300 \mathrm{~s}$ and give its gap if the time limit is reached without a proven optimal solution.

Table 4: Computational results for DP and MILP.

\begin{tabular}{cccccc}
\hline & \multicolumn{5}{c}{ Time horizon (T) } \\
& 20 & 50 & 100 & 200 & 400 \\
\hline DP time & $<0.01 \mathrm{~s}$ & $<0.1 \mathrm{~s}$ & $0.13 \mathrm{~s}$ & $1.5 \mathrm{~s}$ & $22.8 \mathrm{~s}$ \\
MILP gap at DP completion & $0 \%$ & $3.47 \%$ & $32.58 \%$ & $15.27 \%$ & $4.04 \%$ \\
MILP gap after 300 s & $0 \%$ & $2.3 \%$ & $4.23 \%$ & $5.63 \%$ & $3.97 \%$ \\
\hline
\end{tabular}

On one hand we can assert that our DP algorithm is very efficient and its computational time is only dependent on T. Even for 200 periods it takes less than 2 seconds to compute the optimal cost. On the other hand, we have observed that the MILP cannot improve its lower bound easily and that a small improvement requires many nodes to be explored. It is also to be noticed that on the instance presented (except for 20 periods), the MILP ends after $300 \mathrm{~s}$ with an integer solution which is not optimum compared to the cost provided by the DP algorithm.

\section{Conclusion}

We have studied an extension of the constant CLSP with constant batch size under linear and WW cost structures. We have proposed two polynomial time algorithms for the following two cases: production capacity is multiple of the batch size and both capacities can take arbitrary fixed values. Our results generalize that of [5] with the addition of setup costs, however restricted to a constant production capacity and without backlogging.

As a perspective, we may try to improve the time complexity $O\left(T^{6}\right)$ of the algorithm, using Monge arrays as in [3] or polymatroid properties as in [5]. Another perspective can be to extend our results to the backlogging case. Under backlogging 
assumptions, observe that all the properties we introduced still hold, which implies that the inventory level is known modulo $\mathrm{B}$ at the beginning of a sub-interval. However when backlog is allowed, this inventory level can take negative values. It appears that additionnal dominance properties are needed to limit the scope of those negative values and derive polynomial time algorithms. Finally, an important limitation of our algorithm is on the cost assumptions: linear and Wagner-Whitin costs are assumed, as in [5]. The question is open for more general production costs, especially with stepwise concave costs, whether the problem remains polynomial or not with constant production capacities.

\section{Acknoledgments}

We are grateful to the anonymous referees for their careful reading of this paper and their suggestions.

\section{References}

[1] M. Florian, J. Lenstra, A. Rinnooy Kan, Deterministic production planning: Algorithms and complexity, Management Science 26 (7) (1980) 669-679.

[2] G. Bitran, H. Yanasse, Computational complexity of the capacitated lot size problem, Management Science 28 (10) (1982) 1174-1186.

[3] C.-L. Li, V. Hsu, W.-Q. Xiao, Dynamic lot sizing with batch ordering and truckload discounts, Operations Research 52 (4) (2004) 639-654.

[4] A. Akbalik, Y. Pochet, Valid inequalities for the single-item capacitated lot sizing problem with stepwise costs, European Journal of Operational Research 198 (2009) 412-434.

[5] M. Van Vyve, Algorithms for single-item lot-sizing problems with constant batch size, Mathematics of Operations Research 32 (3) (2007) 594613.

[6] A. Manne, Programming of economic lot sizes, Management Science 4 (1958) 115-135.

[7] H. Wagner, T. Whitin, Dynamic version of the economic lot size model, Management Science 5 (1) (1958) 89-96.

[8] M. Florian, M. Klein, Deterministic production planning with concave costs and capacity constraints, Management Science 18 (1) (1971) 1220.

[9] K. Baker, P. Dixon, M. Magazine, E. Silver, An algorithm for the dynamic lot-size problem with time-varying production capacity constraints, Management Science 24 (16) (1978) 1710-1720.

[10] S. Lippman, Optimal inventory policy with multiple set-up costs, Management Science 16 (1) (1969) 118-138.

[11] Y. Pochet, L. Wolsey, Lot-sizing with constant batches: Formulation and valid inequalities, Mathematics of Operations Research 18 (4) (1993) $767-785$.

[12] C.-Y. Lee, A solution to the multiple setup problem with dynamic demand, IIE Transactions 21 (3) (1989) 266-270.

[13] C.-Y. Lee, S. Çetinkaya, W. Jaruphongsa, A dynamic model for inventory lot sizing and outbound shipment scheduling at a third-party warehouse, Operations Research 51 (5) (2003) 735-747.

[14] O. Alp, N. Erkip, R. Gullu, Optimal lot sizing/vehicle dispatching policies under stochastic lead times and stepwise fixed costs, Operations Research 51 (2003) 160-166.

[15] C. van Hoesel, A. Wagelmans, An $O\left(T^{3}\right)$ algorithm for the economic lot-sizing problem with constant capacities, Management Science 42 (1) (1996) 142-150.

[16] Y. Pochet, L. Wolsey, Production Planning by Mixed Integer Programming, Springer, 2006.

[17] A. Manne, A. Veinott, Optimal arbitrary increasing time paths of demand, MIT Press, Cambridge, Massachusetts, 1967, Ch. 11 in A.S. Manne (ed.), Investments for capacity expansion: Size, location and time phasing, pp. 178-190.
[18] A. Akbalik, B. Penz, Exact methods for single-item capacitated lot sizing problem with alternative machines and piece-wise linear production costs, International Journal of Production Economics 119 (2009) 367-379.

\section{Appendix}

The algorithm can be generalized to a special case of time dependent production capacity where $P_{t}=k_{t} B$, for some $k_{t}$ belonging to a fixed set $A$ of integers. Notice that if $A$ is not fixed but part of the instance, the problem is NP-hard (see section 2 ). On the contrary, for a fixed subset $A$, the problem remains polynomial. We give here only the main arguments. Consider $A=\left\{a_{1}, \ldots, a_{q}\right\}$ a fixed subset of integers. For short let us denote by $Q_{j}=a_{j} B$ the $B$-saturated production corresponding to the $j$ th production capacity (such a period is said of type $j$ ). The inventory level at the begining of a sub-interval now depends on our many production periods of each type are saturated till the end of the subplan. More precisely, if period $t$ is, say, of type 1 , given $\vartheta_{j}$ the number of $B$-saturated periods of type $j$ till the end of the subplan for $j \in A \backslash\{1\}$, we can deduce the entering inventory level $\widehat{s}\left(\vartheta_{2}, \ldots, \vartheta_{q}\right)$ of period $k$. Following the principles of section 5.2 it is possible to compute the optimal cost $C^{v}[k, f]\left(\bar{\vartheta}, \bar{\vartheta}^{\prime}\right)$ of a sub-interval $[k, f]$, for given vectors $\bar{\vartheta}$ at period $k$ and $\bar{\vartheta}^{\prime}$ at period $f$, representing the number of $B$ saturated batches of each type till the end of the subplan, excluding the type of period $k$, respectively of period $f$, which can be deduced from flow conservation. This computation is based on the optimal cost $G\left(t, \vartheta_{1}, \vartheta_{2}, \ldots, \vartheta_{q}\right)$ representing the optimal cost for the end of period $t$ till the beginning of period $f$, with exactly $\vartheta_{j}$ batches of type $j$. For given value $v, f$ and $\bar{\vartheta}^{\prime}$ all these quantities $G()$ can be computed in time $T^{|A|+1}$, which gives an overall time complexity in $O\left(T^{(|A|+1)^{2}}\right)$. 